Revue Revue de l'histoire des religions

$$
\begin{array}{cl}
\text { de Ihistoire } & \begin{array}{l}
3 \mid 2013 \\
\text { des religions } \\
\text { Varia }
\end{array}
\end{array}
$$

Giuseppe BUFFon, Sulle tracce di una storia omessa. Storiografia moderna e contemporanea dell'Ordine francescano

Grottaferrata, Frati editori di Quaracchi (« Analecta Franciscana », 18), 2011, 271 p., 27 cm, 35 €, ISBN 978-88-7013-282-3.

Caroline Galland

\title{
OpenEdition
}

Journals

Édition électronique

URL : http://journals.openedition.org/rhr/8148

DOI : $10.4000 /$ rhr.8148

ISSN : 2105-2573

Éditeur

Armand Colin

Édition imprimée

Date de publication : 1 septembre 2013

Pagination : 419-421

ISBN : 978-2200928650

ISSN : 0035-1423

Référence électronique

Caroline Galland, «Giuseppe BufFon, Sulle tracce di una storia omessa. Storiografia moderna e contemporanea dell'Ordine francescano », Revue de l'histoire des religions [En ligne], 3 | 2013, mis en ligne le 04 octobre 2013, consulté le 22 septembre 2020. URL : http://journals.openedition.org/rhr/8148 DOI : https://doi.org/10.4000/rhr.8148

Ce document a été généré automatiquement le 22 septembre 2020.

Tous droits réservés 


\section{Giuseppe BUFFON, Sulle tracce di una storia omessa. Storiografia moderna e contemporanea dell'Ordine francescano}

Grottaferrata, Frati editori di Quaracchi («Analecta Franciscana », 18), 2011, 271 p., 27 cm, 35 €, ISBN 978-88-7013-282-3.

\section{Caroline Galland}

\section{RÉFÉRENCE}

Giuseppe BUFFON, Sulle tracce di una storia omessa. Storiografia moderna e contemporanea dell'Ordine francescano, Grottaferrata, Frati editori di Quaracchi ( Analecta Franciscana », 18), 2011, 271 p., 27 cm, 35 €, ISBN 978-88-7013-282-3.

1 Dans ce nouvel opus, Giuseppe Buffon s'interroge sur le manque d'intérêt pour l'histoire de l'Ordre franciscain à l'époque moderne et contemporaine et propose une étude de l'historiographie franciscaine depuis la rupture officialisée avec les Conventuels en 1517 jusqu'au Concile Vatican II. Ce livre répond à trois objectifs : dresser un état des lieux de la recherche en histoire franciscaine ; fournir des éléments d'explication quant à ce désintérêt et proposer une réflexion sur le franciscanisme comme catégorie historiographique. L'ouvrage ne se limite donc pas à une approche chronologique de l'historiographie franciscaine depuis 1517, il comporte une dimension programmatique. L'enjeu porte précisément sur l'identité franciscaine : l'auteur refuse ainsi la thèse selon laquelle une histoire - et donc une identité - franciscaine n'existerait pas (sur ce point, voir notamment E. L. Furniss, The Franciscan Order in LateMedieval and Early-Modern Western Europe: a Historiographical Survey, 2006) et plaide au contraire pour une identité à rechercher dans l'histoire globale de l'Ordre, aussi complexe et conflictuelle soit-elle.

2 L'ouvrage s'ouvre sur une vaste bibliographie (p. 7-26), point de départ de toute la réflexion. Les sources manuscrites et imprimées portent essentiellement sur deux types 
d'ouvrages : les histoires de l'Ordre écrites depuis le $\mathrm{xvI}^{\mathrm{e}}$ siècle jusqu'au milieu du $\mathrm{xx}^{\mathrm{e}}$ siècle ainsi que ceux qui traitent la question des normes franciscaines. Aux sources s'ajoutent les études sur ce corpus. Il est certain que tel ou tel spécialiste repérera des oublis tant le sujet est large, mais saluons plutôt l'ambition d'une telle bibliographie et le premier état qu'elle livre, sans nul doute fort utile même si elle reste relativement italo-centrée.

3 L'introduction, intitulée « Une catégorie historiographique dépréciée » (p. 29-58), vise à expliquer pourquoi le franciscanisme suscite si peu d'intérêt chez les historiens contemporains. Giuseppe Buffon propose plusieurs facteurs pour expliquer ce désert historiographique dont on retiendra les deux principaux. Tout d'abord, le contexte du Concile Vatican II. S'il est vrai que les ordres religieux furent incités à ce moment-là à lancer des études internes pour mieux connaître leurs origines, leurs fondateurs et leurs grands réformateurs, ils eurent tendance à mal interpréter ces injonctions en privilégiant le moment des origines stricto sensu. Or, la question des origines constitue pour les Franciscains un véritable problème historiographique (voir sur ce point $\mathrm{S}$. da Campagnola, Le origini francescane come problema storiografico, 1979). À cet élément d'explication s'en ajoute un autre, tout aussi important: le développement des études sur le Concile de Trente - avec le passage de la Contre-Réforme à la "Réforme catholique " initié par Hubert Jedin - qui privilégia les études sur les évêques et négligea, parallèlement, la place des religieux dans l'histoire post-tridentine. Mais les récentes orientations historiographiques n'expliquent pas tout et l'auteur se demande pourquoi, en général, les institutions de l'Ordre ont préféré, depuis la fin du XIXe siècle, l'histoire des origines à celle de la durée, l'idéal de la première heure aux évolutions complexes. On ne saurait trop recommander la lecture de ces pages aux novices de l'histoire franciscaine tant les problèmes historiographiques sont bien posés. Giuseppe Buffon retrace notamment l'histoire récente d'un concept herméneutique, «la rupture ", depuis Heribert Holzapfel - qui écrivit son histoire de l'Ordre pour appuyer la bulle léonine de 1897 visant à redonner une unité à l'Observance - jusqu'à Kajetan Esser - qui défendait cinquante ans après le point de vue de Holzapfel en souhaitant revenir à la pureté originelle de la Règle et éliminer les interprétations multiples de la Règle. À ce courant interprétatif, l'auteur oppose un Théophile Desbonnets qui proposait une herméneutique différente de la fracture, non pas tant entre les origines et le développement mais plutôt entre l'intuition et l'institutionnalisation.

Dans la première partie, intitulée «Des débats sur la pauvreté à la légitimation institutionnelle » (p. 59-128), Giuseppe Buffon revient sur les multiples effets de la bulle Ite Vos dans l'historiographie franciscaine, de 1517 jusqu'en 1688 - date de la publication d'une histoire de l'Ordre par le ministre général des Conventuels, Casimir Biernacki. L'auteur montre bien comment le pluralisme interprétatif - les différentes lectures de la Règle et notamment de la pauvreté - aboutit à un pluralisme institutionnel - les Conventuels, les Observants puis les Capucins - qui lui-même engendre de nouvelles orientations historiographiques. L'enjeu, à partir de 1517, n'est plus tant de discuter ni de justifier telle ou telle lecture de la Règle mais plutôt, pour chaque branche, d'avancer les arguments de sa légitimité. Pour illustrer ce basculement, l'auteur étudie avec précision des histoires globales de l'Ordre produites par des historiens issus des différentes branches de la famille franciscaine: un Observant de la première génération d'après la bulle (Marc de Lisbonne qui écrit sa Cronaca entre 1557 et 1570), un Conventuel formé à l'école humaniste (Pierre Ridolfi et 
son Historia seraphica en 1585), un ministre général Observant (François de Gonzague et son De origine seraphicae religionis en 1587), des Capucins (Bernardin Croli de Colpetrazzo (1579), Matthias Bellintani de Salò (1587) et Zacharie Boverio (1632), un annaliste du $\mathrm{XVII}^{\mathrm{e}}$ siècle caractéristique de l'historiographie ecclésiastique érudite de l'âge baroque (l'incontournable Luc Wadding et ses Annales Minorum (1625-1654), des auteurs de manuels d'histoire franciscaine (François Harold (1662), le neveu de Luc Wadding, et Diego Tafuro da Lequile (1664) et enfin un annaliste conventuel de la fin du XVII siècle (Casimir Biernacki). Dans chacune de ces histoires de l'Ordre, l'auteur se demande comment la scission de 1517 est perçue, interprétée et au final vécue. Pour les Capucins par exemple, le thème récurrent de la décadence des Observants se double d'une périodisation de l'histoire de l'Ordre : la dernière des réformes - en l'occurrence celle des Capucins - est présentée comme la plus authentique et la plus parfaite. Dans cette analyse, l'auteur n'oublie pas d'identifier les autorités mobilisées: alors que les Observants de la fin du $\mathrm{XvI}^{\mathrm{e}}$ siècle continuent de se référer à Bartolomé de Pise, les Capucins préfèrent en appeler au "schéma apocalyptique » des Spirituels Ange Clareno et Ubertin de Casale. Enfin, cette étude des histoires de l'Ordre accorde une attention particulière à la question du genre littéraire, opérant une fine distinction entre les chroniques, les annales ou encore les manuels d'histoire.

5 C'est d'ailleurs le fil qui est déroulé dans la seconde partie de l'ouvrage, intitulée «Entre auteurs et genres littéraires » (p. 130-228). Plus complexe, cette partie n'étudie plus les histoires globales mais distingue six types d'histoires particulières: les histoires littéraires, les hagiographies, les manuels d'histoire à visée didactique, les ouvrages polémiques, les histoires des missions ou plutôt les histoires qui tendent à penser l'Ordre dans une optique missionnaire, les histoires de la hiérarchie de l'ordre. Pour chaque type d'histoire, l'auteur s'intéresse à la manière dont les différentes branches présentent et pensent l'Ordre dans sa globalité. Giuseppe Buffon se demande par exemple dans quelle mesure les auteurs d'histoires littéraires ont su intégrer l'ensemble des écrivains franciscains. Un premier pas est franchi avec Luc Wadding dans son Scriptores Ordinis Minorum (1650) qui reprend un genre inauguré par les Conventuels dans la tradition humaniste et érudite en lui donnant des accents universalistes. Néanmoins, d'après l'auteur seuls les Capucins ont su effectuer le passage du genre des Scriptores à celui de la bibliographie, en fondant notamment une revue qui ne se limite pas au champ strictement capucin. Ce point ne manquera pas de susciter de vifs débats, au moins chez les Frères Mineurs.

6 Dans la troisième partie sobrement intitulée «Paradigme » et plus courte que les autres (p. 229-254), Giuseppe Buffon revient sur l'histoire de l'Ordre écrite en 1909 par H. Holzapfel. Il en propose une analyse extrêmement fine qui permet de saisir tous les enjeux : dans cette version de l'histoire, les réformes conduisent à une prolifération des formes d'autonomie et donc à la formation de groupes de pouvoirs aux effets délétères. Mais si l'auteur s'oppose aux résultats de Holzapfel, il n'en salue pas moins la démarche: chercher à esquisser une grille de lecture pour comprendre dans son ensemble le phénomène franciscain. C'est également l'objectif formulé par K. Esser dans son Memorandum écrit après le chapitre général post-conciliaire de 1967: «L'Ordre a besoin d'une nouvelle histoire ». Mais là encore, le paradigme ne doit pas être celui d'une condamnation des réformes.

7 C'est donc dans la conclusion (p. 257-259) que l'auteur livre véritablement son point de vue: la spécificité de l'identité franciscaine pourrait ou devrait se lire à travers 
l'analyse de sa "complexité institutionnelle». L'historien insiste en effet sur la nécessité de prendre en compte, notamment pour l'époque moderne, l'histoire officielle de l'Ordre mais aussi les histoires particulières, celles produites par les ordres réformés. Pour saisir la "modernité », il est fondamental de comprendre l'identité conflictuelle et complexe $\mathrm{du}$ franciscanisme dont l'historiographie témoigne sans ambages.

\section{AUTEURS}

\section{CAROLINE GALLAND}

Université Paris Ouest - Nanterre La Défense. 